\title{
Erratum to: Speech Authentication and Recovery Scheme in Encrypted Domain
}

\author{
Qing Qian, Hongxia Wang ${ }^{(\bowtie)}$, Sani M. Abdullahi, Huan Wang, \\ and Canghong Shi \\ School of Information Science and Technology, \\ Southwest Jiaotong University, Chengdu 611756, China \\ hxwang@swjtu.edu.cn
}

\section{Erratum to: \\ Chapter "Speech Authentication and Recovery \\ Scheme in Encrypted Domain" in: Y.Q. Shi et al. (Eds.): \\ Digital Forensics and Watermarking \\ DOI: 10.1007/978-3-319-53465-7_4}

By mistake the name of the third author - Sani M. Abdullahi - was misspelled in the original version.

\footnotetext{
The updated original online version for this chapter can be found at DOI: $10.1007 / 978-3-319-53465-7 \_4$ 